\title{
Evolutionary Shift from Purifying Selection towards Divergent Selection of SARS-CoV2 Favors its Invasion into Multiple Human
} Organs

\author{
Amit K Maiti ${ }^{1}$ \\ ${ }^{1}$ Department of Genetics and Genomics
}

April 29, 2021

\begin{abstract}
SARS-CoV2 virus is believed to be originated from a closely related bat Coronavirus RaTG13 lineage after gaining insertions of RBD of spike (S) protein by exchanged recombination with pangolin virus Pan_SL_COV_GD. SARSCoV2 uses its entry-point key residues in S1 protein to attach with human ACE2 receptor. SARS-CoV2 evolution comprises any of these possibilities: it entered human from bat with its poorly developed entry-point residues much before its known appearance with slower mutation rate; or recently with efficiently developed entry-point residues having more infective power with higher mutation rate; or through an intermediate host. RaTG13 has 96.3\% identity with SARS-CoV2 genome implying that it substituted 1106 nucleotides to evolute as present-day virus. Temporal analysis of SARS-CoV2 genome from December 2019 shows that its nucleotide substitution rate is as low as $27 \mathrm{nt} /$ year with an evolutionary rate of 9x10-4 / site/year, which is a little less than other retrovirus (10-4 to 10-6 /site/year). Estimation of TMRCA of SARS-CoV2 from bat RaTG13 lineage appears to be in between 9-14 years. Furthermore, evolution of a critical entry-point residue Y493Q needs two substitutions with an intermediate virus carrying $\mathrm{Y} 493 \mathrm{H}(\mathrm{Y}>\mathrm{H}>\mathrm{Q})$, although such an intermediate virus has not been identified in known twenty-nine bat CoV virus. Genetic codon analysis indicates that SARS-CoV2 evolution from RaTG13 lineage strictly follows neutral evolution with strong purifying selection whereas its propagation in human disobeys neutral evolution as nonsynonymous mutations surpasses synonymous mutations with the increase of $\omega(\mathrm{dn} / \mathrm{ds})$ signifying its proceedings towards divergent selection predictably for its infection power to evade multiple organs.
\end{abstract}

\section{Hosted file}

corona-evolution paper-autarea.pdf available at https://authorea.com/users/337405/articles/ 520015-evolutionary-shift-from-purifying-selection-towards-divergent-selection-of-sarscov2-favors-its-invasion-into-multiple-human-organs 